\title{
Predictive and prognostic value of prognostic nutritional index for locally advanced breast cancer
}

\author{
Yaohui Wang ${ }^{1 \#}$, Bayarmaa Battseren ${ }^{1 \#}$, Wenjin Yin ${ }^{1}$, Yanping Lin ${ }^{1}$, Liheng Zhou ${ }^{1}$, Fan Yang ${ }^{1}$, Yan Wang ${ }^{1}$, \\ Lu Sun ${ }^{2}$, Jinsong Lu ${ }^{1}$ \\ ${ }^{1}$ Department of Breast Surgery, Renji Hospital, School of Medicine, Shanghai Jiaotong University, Shanghai 200127, China; ${ }^{2}$ Department of \\ Gynecological Oncology, Zhejiang Cancer Hospital, Hangzhou 310022, China \\ Contributions: (I) Conception and design: Y Wang, J Lu; (II) Administrative support: J Lu; (III) Provision of study materials or patients: All authors; \\ (IV) Collection and assembly of data: B Battseren, Y Lin, L Zhou, F Yang, Y Wang, L Sun; (V) Data analysis and interpretation: Y Wang, W Yin; (VI) \\ Manuscript writing: All authors; (VII) Final approval of manuscript: All authors. \\ \#These authors contributed equally to this work. \\ Correspondence to: Jinsong Lu. Department of Breast Surgery, Renji Hospital, School of Medicine, Shanghai Jiaotong University, No. 1630 Dongfang \\ Road, Shanghai 200127, China. Email: lujjss@163.com.
}

\begin{abstract}
Background: The prognostic nutritional index (PNI) is an indicator of nutritional immune status. Recently, the PNI has been found to be significantly associated with the clinical outcome of various solid tumors. Few patients with newly diagnosed breast cancer are in a state of malnutrition. In contrast, breast cancer is usually an overnutrition-related disease. This study aimed to explore the relationship of an excessively high PNI with sensitivity to neoadjuvant therapy and the prognosis of patients with locally advanced breast cancer.
\end{abstract}

Methods: A total of 202 patients from two clinical trials, SHPD002 and SHPD003, were included. Binary logistic regression analysis was used to assess the association between the PNI and pathological complete response (pCR). Univariate and multivariate survival analyses were performed to assess the prognostic factors used to predict disease-free survival (DFS).

Results: An excessively high PNI was more difficult to achieve pCR (OR =0.322; 95\% CI, 0.132-0.788, $\mathrm{P}=0.013$ ) and was associated with a worse DFS (log-rank $\mathrm{P}=0.013)$. The $\mathrm{PNI}$ was an independent prognostic factor for DFS in all patients $(\mathrm{HR}=3.027$; $95 \% \mathrm{CI}, 1.207-7.592, \mathrm{P}=0.018)$, the premenopausal $(\mathrm{HR}=8.292$; 95\% CI, 1.670-41.17, P=0.010), clinical T3 and T4 (HR =3.405; 95\% CI, 1.141-10.16, P=0.028), ER negative (HR =9.698; 95\% CI, 1.205-78.07, $\mathrm{P}=0.033)$, HER2 negative (HR =3.765; 95\% CI, 1.101-12.88, $\mathrm{P}=0.035)$ and $\mathrm{pCR}$ subgroups ( $\mathrm{HR}=11.912$; $95 \% \mathrm{CI}, 1.326-107.0, \mathrm{P}=0.027$ ).

Conclusions: An excessively high PNI was a risk factor for sensitivity to neoadjuvant therapy and prognosis of patients with locally advanced breast cancer.

Keywords: Prognostic nutritional index (PNI); breast neoplasms; neoadjuvant therapy; therapeutic index; prognosis

Submitted Jul 17, 2019. Accepted for publication Oct 08, 2019.

doi: $10.21037 /$ gs.2019.10.08

View this article at: http://dx.doi.org/10.21037/gs.2019.10.08

\section{Introduction}

Nutritional immune status is closely related to various aspects of malignant tumors. Previous studies have found that malnutrition and poor immune status might increase the risk of postoperative complications, reduce the response to antitumor therapy, and be related to poor survival (1-4).

The prognostic nutritional index (PNI) was initially used to evaluate nutritional status in patients undergoing surgery (5). Onodera et al. simplified the algorithm of the PNI which was calculated with serum albumin and total peripheral 


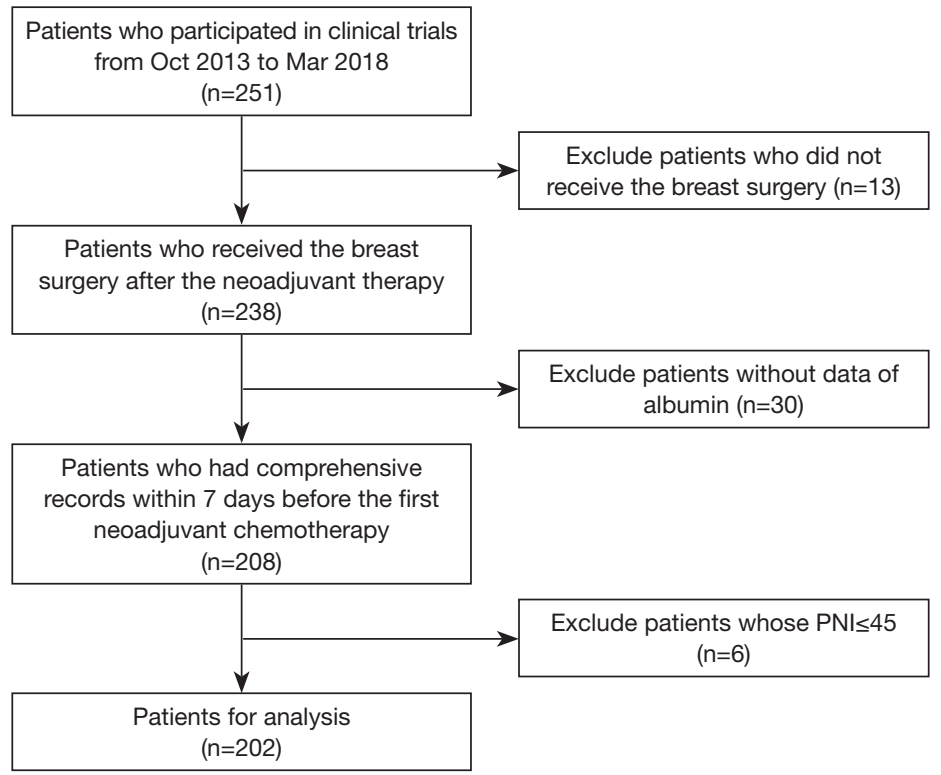

Figure 1 Diagram of patient selection.

blood lymphocytes (6). Currently, the PNI has been extended to the field of cancer as an indicator of nutritional immune status and has been found to be significantly associated with the therapeutic effects and long-term prognosis of various solid tumors (7-10).

In gastrointestinal tumors, low PNI was an independent poor prognostic factor for patients $(7,8,10)$. Breast cancer is the most common cancer in the world, and seriously endangers women's health (11). A relatively low PNI was also found to be related to poor clinical outcome of breast cancer (12). However, fewer breast cancer patients in the early stage or even in the locally advanced stage showed significant malnutrition at the time of diagnosis. Instead, breast cancer might commonly be an overnutritionrelated disease. Obesity is a vital risk factor for breast cancer in postmenopausal women (13). For breast cancer patients, a higher body mass index (BMI) was related to poor prognosis and resistance to therapy $(14,15)$. Previous studies have shown a significant positive correlation between PNI and BMI (16). Collectively, we hypothesized that an excessively high PNI may be associated with poor chemotherapy response and poor prognosis in breast cancer patients. To test this hypothesis, we retrospectively analyzed the relationship between an excessively high PNI and the treatment efficacy and prognosis of patients with locally advanced breast cancer who received neoadjuvant therapy.

\section{Methods}

\section{Patients and treatment}

This study was a retrospective analysis based on prospective clinical trials, of SHPD002 (NCT02221999) and SHPD003 (NCT02879513), conducted in Renji Hospital, School of Medicine, Shanghai Jiao Tong University. Forty-five is a widely used cutoff of PNI in the context of gastrointestinal surgery (6) due to the number of studies that regarded a PNI of less than or equal to 45 as low and the fact that low PNI was highly associated with poor prognosis of cancer patients $(4,9)$ We excluded patients with a PNI less than or equal to 45 and explored the prognostic and predictive value of the PNI above safe surgery cutoff (PNI >45). A total of 251 patients participated in these two clinical trials from October, 2013 to March, 2018. Thirteen patients who did not undergo breast surgery after neoadjuvant therapy were dropped, and five of them were lost to follow-up. Thirty patients who did not have a comprehensive record of complete blood count and albumin within 7 days before the first round of neoadjuvant chemotherapy were excluded. Six patients' PNI values were less than or equal to 45 were also excluded. Thus, 202 patients were analyzed in this study (Figure 1). All patients received weekly paclitaxel with cisplatin for 16 weeks before the surgery. Patients with positive hormone receptors randomly either combined 
the chemotherapy with neoadjuvant endocrine therapy (letrozole for postmenopausal patients and leuprorelin or goserelin for premenopausal patients) or not. Human epidermal growth factor receptor 2 (HER2)-positive patients received trastuzumab concurrent with neoadjuvant therapy for one year. After surgery, if the patients did not achieve the pathological complete response (pCR), they randomly received two cycles of weekly paclitaxel with cisplatin or four cycles of cyclophosphamide, epirubicin and fluorouracil. If the patients achieved the pCR, they randomly received two cycles of weekly paclitaxel with cisplatin or no more chemotherapy. The use of adjuvant radiotherapy was decided by the radiologist. Patients who were hormone receptor-positive received adjuvant endocrine therapy. Telephone follow-up or outpatient visits should be conducted every 3 months. All procedures implemented in the studies involving human participants conformed to the ethical standards of the institutions and national research committees, as well as the 1964 Declaration of Helsinki and its subsequent amendments or similar ethical standards. The study was approved by the Institutional Review Board (IRB) of Renji Hospital, School of Medicine, Shanghai Jiao Tong University \{IRB approval number: [2017]088\}. Written informed consent was provided by all patients. This analysis was designed and the results were reported in accordance with the Reporting Recommendations for Tumor Marker Prognostic Studies (REMARK) guidelines (17).

\section{Data collection}

Basic information which included age, menopausal status, height, weight and tumor information which consisted of tumor stage, estrogen receptor (ER) status, progesterone receptor (PR) status, HER2 receptor status, and ki67 index of the primary tumor before neoadjuvant therapy, was collected. ER and PR expression greater than or equal to $1 \%$ was defined as positive and less than $1 \%$ was defined as negative. ER or PR positivity was defined as hormone-receptor positive. ER and PR negativity were defined as hormone-receptor negative. FISH amplification or immunohistochemistry of HER2 3+ was defined as HER2 positive. No invasive breast cancer in the breast and no pathological involvement in the axillary lymph nodes were defined as pCR. Blood tests, including serum albumin and total peripheral blood lymphocytes, were performed within 7 days before the first round of neoadjuvant chemotherapy and were recorded to calculate the PNI. The PNI is equal to $10 \times$ the serum albumin concentration $(\mathrm{g} / \mathrm{dL})+0.005 \times$ the total lymphocyte count $\left(\right.$ per $\left.\mathrm{mm}^{3}\right)$. The upper quartiles of all patients' PNI was 55. Thus, the definition of an excessively high PNI was a PNI greater than or equal to 55 , and a high PNI was greater than 45 and less than 55 .

\section{Statistical methods}

The correlations of the PNI with the clinicopathological categorical variables were determined using $\chi^{2}$ test. Univariate and multivariate analyses using a binary logistic regression model were performed to analyze the association of pCR with PNI or clinicopathological variables. Odds ratios (ORs) and 95\% confidence intervals (CIs) are shown. Variables considered in the multivariate model of predicting sensitivity of pCR and survival analysis included menopausal status, tumor stage, ER status, PR status, HER2 status and ki67 index. There was no collinearity between each variable. Disease-free survival (DFS) was defined as the time between the operation and the first recurrence of locoregional relapse, distant metastasis, contralateral breast cancer, other second primary cancer or death from any cause. Patients who were still alive and event-free at the last follow-up were censored. The Kaplan-Meier method and log-rank test were used for survival analysis. Cox proportional hazard regression analyses were performed to investigate the association of PNI or each variable with DFS. Hazard ratios (HRs) and 95\% CIs were calculated. A P value $<0.05$ was identified as statistically significant. Stata 14.0 was used for data analysis. (Stata Corporation, College Station, TX, USA).

\section{Results}

\section{Clinicopathologic characteristics}

A total of 202 patients were included in the analyses, with a median follow-up of 26 months (IQR, 16-42 months). One hundred forty-seven patients were in the high-PNI group and 55 patients were in the excessively-high-PNI group. The median Ki67 index was $40 \%$ in high-PNI, excessivelyhigh-PNI group and total patients. There were 65 patients who achieved a pCR, and the total pCR rate was $32.18 \%$. In terms of menopausal status $(\mathrm{P}=0.350)$, clinical tumor stage $(\mathrm{P}=0.575)$, ER status $(\mathrm{P}=0.189)$, $\mathrm{PR}$ status $(\mathrm{P}=0.215)$, HER2 status $(\mathrm{P}=0.847)$, the high-PNI and excessively-high-PNI groups were not significantly different (Table 1). 
Table 1 Patient characteristics

\begin{tabular}{|c|c|c|c|c|}
\hline \multirow[b]{2}{*}{ Variables } & \multirow{2}{*}{$\begin{array}{l}\text { Total patients } \\
\begin{array}{c}(\mathrm{N}=202), \mathrm{n} \\
(\%)\end{array}\end{array}$} & \multicolumn{2}{|c|}{ PNI } & \multirow[b]{2}{*}{$\mathrm{P}$} \\
\hline & & $\begin{array}{c}\text { High } \\
(\mathrm{N}=147)\end{array}$ & $\begin{array}{l}\text { Excessively } \\
\text { high }(\mathrm{N}=55)\end{array}$ & \\
\hline \multicolumn{5}{|l|}{ Menopausal status } \\
\hline Premenopausal & 99 (49.01) & 75 & 24 & 0.350 \\
\hline Postmenopausal & 103 (50.99) & 72 & 31 & \\
\hline \multicolumn{5}{|l|}{ Clinical T stage } \\
\hline T1-T2 & $100(49.50)$ & 71 & 29 & 0.575 \\
\hline T3-T4 & $102(50.50)$ & 76 & 26 & \\
\hline \multicolumn{5}{|l|}{ ER status } \\
\hline Negative & 63 (31.19) & 42 & 21 & 0.189 \\
\hline Positive & $139(68.81)$ & 105 & 34 & \\
\hline \multicolumn{5}{|l|}{ PR status } \\
\hline Negative & $50(24.75)$ & 33 & 17 & 0.215 \\
\hline Positive & $152(75.25)$ & 114 & 38 & \\
\hline \multicolumn{5}{|l|}{ HER2 status } \\
\hline Negative & $119(58.91)$ & 86 & 33 & 0.847 \\
\hline Positive & 83 (41.09) & 61 & 22 & \\
\hline \multicolumn{5}{|l|}{$\mathrm{pCR}$} \\
\hline No & $137(67.82)$ & 95 & 42 & 0.112 \\
\hline Yes & 65 (32.18) & 52 & 13 & \\
\hline
\end{tabular}

PNI, prognostic nutrition index; ER, estrogen receptors; PR, progesterone receptors; HER2, human epidermal growth factor receptor 2; pCR, pathological complete response.

\section{Association of PNI and pCR}

In univariate logistic regression analysis, negative ER status (OR $=0.198 ; 95 \%$ CI, 0.104-0.377, $\mathrm{P}<0.001)$, positive HER2 status $(\mathrm{OR}=3.498$; 95\% CI, 1.888-6.478, $\mathrm{P}<0.001)$ and a higher Ki67 index (OR =1.042; 95\% CI, 1.026-1.058, $\mathrm{P}<0.001)$ favored $\mathrm{pCR}$. Menopausal status ( $\mathrm{OR}=1.081 ; 95 \%$ CI, 0.599-1.951, $\mathrm{P}=0.796)$, clinical tumor stage $(\mathrm{OR}=0.644$; 95\% CI, 0.355-1.168, $\mathrm{P}=0.147), \mathrm{PR}$ status $(\mathrm{OR}=0.563$; 95\% CI, 0.089-1.091, $\mathrm{P}=0.089)$, and $\mathrm{PNI}(\mathrm{OR}=0.565 ; 95 \%$ CI, $0.279-1.148, \mathrm{P}=0.115$ ) were not found to be obviously associated with pCR.

After adjusting for menopausal status, clinical tumor stage, ER status, PR status, HER2 status and Ki67 index, the multiple logistic regression analysis revealed that excessively high PNI was significantly associated with the non-pCR group (OR $=0.322 ; 95 \%$ CI, 0.132-0.788, $\mathrm{P}=0.013)$. Patients with a higher Ki67 index achieved pCR more easily ( $\mathrm{OR}=1.039 ; 95 \% \mathrm{CI}, 1.020-1.058, \mathrm{P}<0.001$ ). In addition, $\mathrm{ER}$ status $(\mathrm{OR}=0.152 ; 95 \% \mathrm{CI}, 0.059-0.389$, $\mathrm{P}<0.001)$ and HER2 status $(\mathrm{OR}=4.591 ; 95 \% \mathrm{CI}, 2.126-$ 9.917, $\mathrm{P}<0.001$ ) could also predict pCR (Table 2).

\section{Survival analyses}

In the univariate analysis, the DFS of the excessively-highPNI group was significantly shorter than that of the highPNI group (log-rank $\mathrm{P}=0.013$, Figure 2). Multivariate analysis showed that the PNI was an independent prognostic factor for DFS (HR =3.027; 95\% CI, 1.207-7.592, P=0.018,

Table 2 Univariate and multivariate binary logistic regression analyses of PNI or clinical characteristics and pathological complete response to neoadjuvant therapy

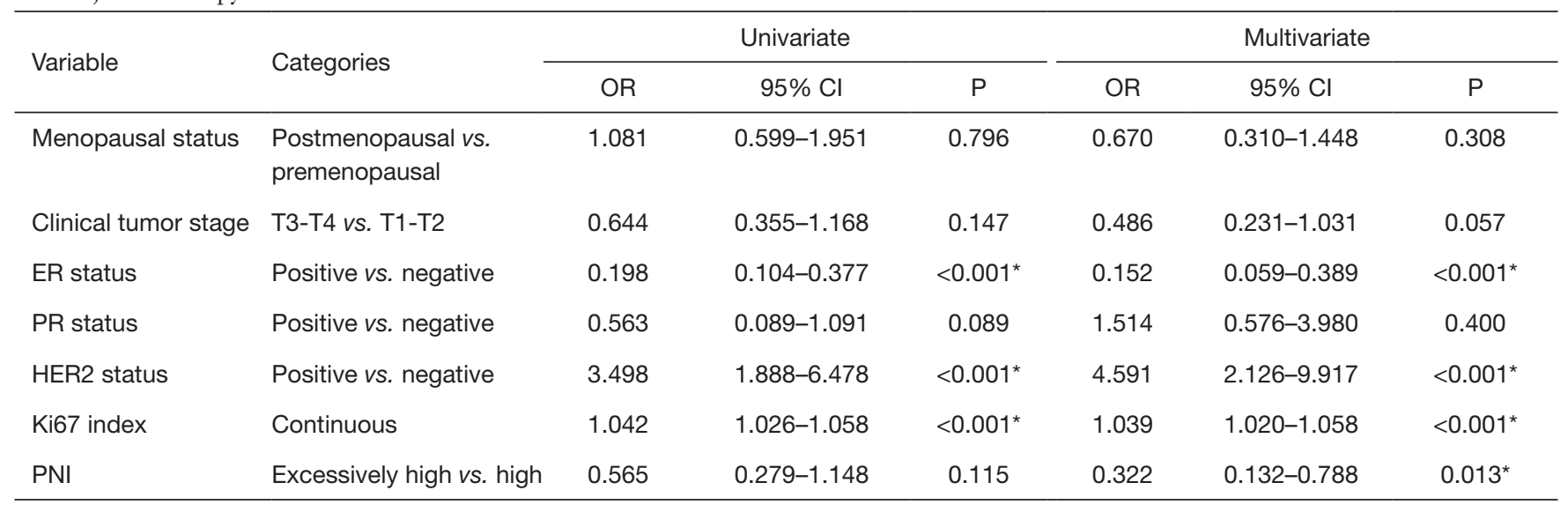

*, indicates statistical significance $(\mathrm{P}<0.05)$. ER, estrogen receptor; PR, progesterone receptor; HER2, human epidermal growth factor receptor 2; PNI, prognostic nutrition index; OR, odds ratio; $\mathrm{Cl}$, confidence interval. 


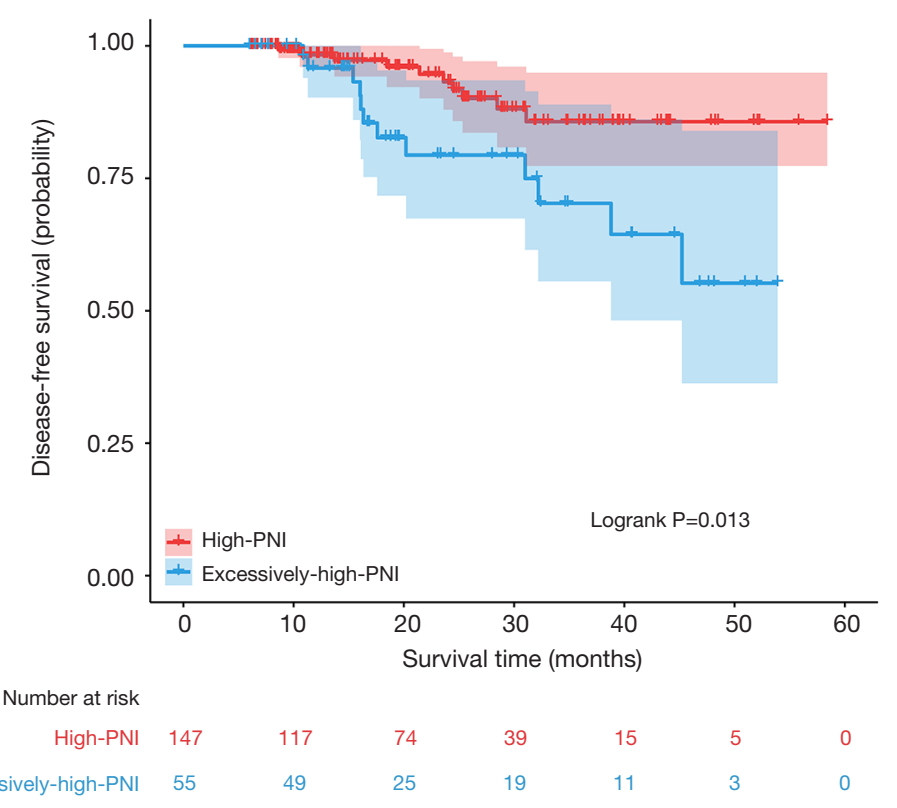

Figure 2 Kaplan-Meier survival curve for disease-free survival in the high-PNI group versus the excessively-high-PNI group. PNI, prognostic nutrition index.

Table 3 Cox proportional hazards regression: multivariate survival analysis of PNI and disease-free survival

\begin{tabular}{llcc}
\hline Variable & Categories & HR & 95\% Cl \\
\hline Menopausal status & Postmenopausal vs. premenopausal & 1.608 & $0.632-4.093$ \\
Clinical tumor stage & T3-T4 vs. T1-T2 & 1.474 & $0.613-3.545$ \\
ER status & Positive vs. negative & 1.209 & 0.319 \\
PR status & Positive vs. negative & 0.524 & 0.486 \\
HER2 status & Positive vs. negative & 1.385 & $0.200-1.428$ \\
Ki67 index & Continuous & 1.028 & $0.591-3.246$ \\
PNI & Excessive high vs. high & 3.027 & $1.007-1.050$ \\
\hline
\end{tabular}

*, indicates statistical significance $(\mathrm{P}<0.05)$. ER, estrogen receptor; PR, progesterone receptor; HER2, human epidermal growth factor receptor 2; PNI, prognostic nutrition index; HR, hazard ratio; $\mathrm{Cl}$, confidence interval.

Table 3). In addition, in the premenopausal $(\mathrm{HR}=8.292$; 95\% CI, 1.670-41.17, $\mathrm{P}=0.010$ ), clinical T3 and T4 (HR $=3.405 ; 95 \% \mathrm{CI}, 1.141-10.16, \mathrm{P}=0.028)$, ER negative $(\mathrm{HR}$ $=9.698 ; 95 \%$ CI, $1.205-78.07, \mathrm{P}=0.033)$, HER 2 negative $(\mathrm{HR}=3.765 ; 95 \% \mathrm{CI}, 1.101-12.88, \mathrm{P}=0.035)$ and $\mathrm{pCR}$ subgroups $(\mathrm{HR}=11.912 ; 95 \% \mathrm{CI}, 1.326-107.0, \mathrm{P}=0.027$, Figure 3), the PNI was also significantly related to the DFS of patients.

\section{Discussion}

In this study, we evaluated the relationship between the PNI and the treatment sensitivity and prognosis for breast cancer patients who received neoadjuvant chemotherapy. As far as we know, this was the first time that patients with excessively-high PNI were found to have more difficultly achieving pCR and to have shorter DFS durations. 


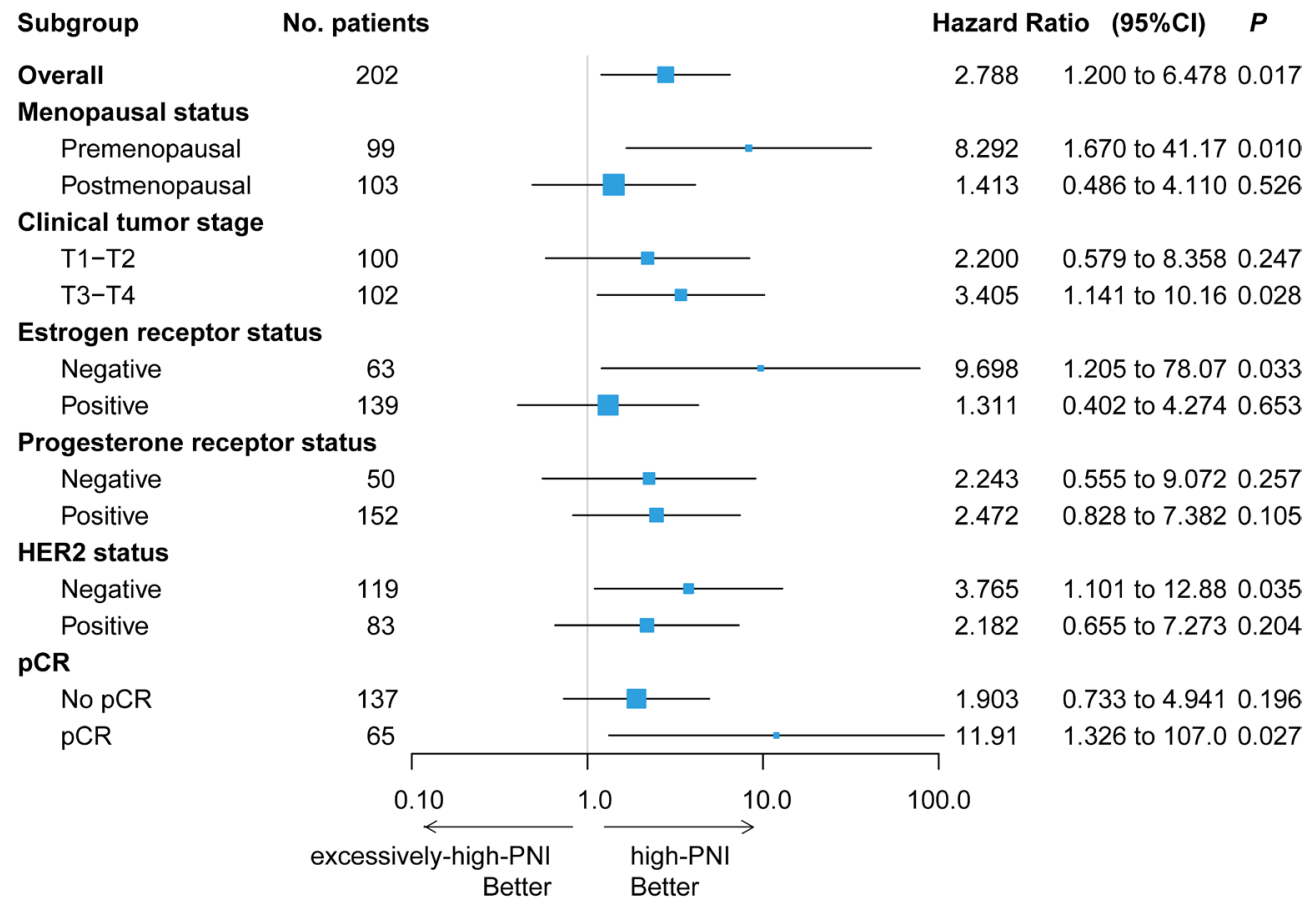

Figure 3 Forest plot of disease-free survival. Subgroup analysis for disease-free survival were performed using patient baseline characteristics.

Therefore, we propose a new viewpoint that an excessively high PNI is a risk factor for poor treatment efficacy and prognosis for breast cancer patients undergoing neoadjuvant chemotherapy. Given that nutritional immune status of cancer patients has attracted increasing attention, our research has important clinical implications.

The PNI is a serum albumin- and peripheral blood lymphocyte-based nutritional parameter (6). The PNI was initially used to provide an accurate, quantitative estimate of operative risk in individuals undergoing gastrointestinal surgery (5). Currently, the PNI is a simple indicator that has extensive clinical applications. The relationship between the PNI and prognosis in various types of malignancies was reported previously $(4,10,16,18,19)$. Although findings related to the PNI and prognosis have been reported in breast cancer patients $(12,20)$, these studies did not focus on the difference between high and excessively high PNI values. Because U-shaped or J-shaped associations have been found between BMI and all-cancer mortality $(21,22)$, we postulated that both low PNI and excessively high PNI might predict poor prognosis in breast cancer patients. Fewer patients who were initially diagnosed with breast cancer had a lower PNI. Out of 208 patients with comprehensive records in our study, only 6 patients'
PNI was less than or equaled to 45 . In contrast to the abovementioned prior research, we excluded patients with low PNI values (PNI $\leq 45)$ and divided patients into highPNI and excessively-high-PNI group. Furthermore, we tested our hypothesis using a prospective follow-up database of locally advanced breast cancer patients in neoadjuvant clinical trials and found that patients with excessively high PNI values had significantly shorter DFS than patients with high PNI values. An excessively high PNI was an independent risk factor for DFS among nonmalnourished neoadjuvant chemotherapy patients. Several studies have found that nutritional status was related to the prognosis of breast cancer $(14,15,23,24)$. A meta-analysis that included data from 213,075 breast cancer patients in 82 studies (including 23,182 cases of breast cancer-related deaths) suggested that overweight (BMI 25-30, RR $=1.07 ; 95 \%$ CI, 1.02-1.12) and obesity (BMI $>30, \mathrm{RR}=1.41 ; 95 \% \mathrm{CI}$, 1.29-1.53) increased the risk of breast cancer death (24). In patients who received neoadjuvant therapy, high BMI was associated with worse DFS and overall survival (OS) (23). All these data indirectly supported our results. It was reported that obesity caused chronic inflammation both systemically and locally and promoted breast cancer growth $(25,26)$. However, inflammation and its protumor 
consequences could be found in some individuals who are not considered obese or overweight by BMI (27). Thus, the PNI is a good complement to BMI for the estimation of the overnutrition status and prognosis of breast cancer patients.

Nutritional parameters have been reported to be related to sensitivity to treatment. Previous studies have shown that overnutrition could detrimentally affect treatment outcomes (23). In the Arimidex, Tamoxifen Alone or in Combination (ATAC) trial, Sestak et al. found that obese patients $\left(B M I>35 \mathrm{~kg} / \mathrm{m}^{2}\right)$ had a higher recurrence rate in a group of postmenopausal breast cancer patients treated with anastrozole or tamoxifen. The relative efficacy of anastrozole compared to tamoxifen was greater in the thin postmenopausal women (28). In terms of neoadjuvant therapy, Fontanella et al. combined data from 8,872 patients in eight neoadjuvant clinical trials of breast cancer and performed an analysis. A BMI $>30 \mathrm{~kg} / \mathrm{m}^{2}$ had a negative impact on pCR rate (23). In our study, we also found that a higher BMI $(\geq 24)$ might make it more difficult to achieve pCR ( $\mathrm{P}=0.049)$. The PNI, which combines albumin and lymphocyte count, might reflect nutritional and immune status. A higher PNI might indicate a better nutritional status. Consistent with the BMI results, the patients in our study with an excessively high PNI (>55) had more difficultly to achieve pCR. Our results demonstrated that an excessively high PNI could predict poor treatment response and further explored the meaning of the PNI in the context of breast cancer. In addition, many large prospective neoadjuvant clinical trials have demonstrated that patients who achieved pCR might have a better long-term prognosis $(29,30)$. Our study found that patients with an excessively high PNI had more difficultly achieving pCR than patients with a high PNI. Therefore, this finding could partially explain the poor prognosis of patients with an excessively high PNI.

The major strengths of this study were that it was based on neoadjuvant clinical trials and that the follow-up database was perspective. The limitations were the relatively small sample size, the short mean follow-up duration and the current absence of OS results. The OS will be analyses after a longer follow-up. Further validation in a large prospective study is needed to further assess the predictive and prognostic value of PNI for breast cancer patients in the future.

\section{Conclusions}

Overall, an excessively high PNI was a risk factor for sensitivity to neoadjuvant therapy and prognosis of patients with locally advanced breast cancer. Large-scale, prospective studies are needed in the future to further validate and optimize the predictive power of the PNI in terms of neoadjuvant chemotherapy for breast cancer.

\section{Acknowledgments}

Funding: This work was supported by the National Natural Science Foundation of China (grant numbers 81172505, 81302302), the Shanghai Municipal Commission of Health and Family Planning (grant number 201640006), the Clinical Research Plan of SHDC (grant number 16CR3065B, grant number SHDC 12016231), the Nurturing Fund of Renji Hospital (grant number PYMDT-002, grant number PY2018-IIC-01), the Shanghai "Rising Stars of Medical Talent" Youth Development Program Youth Medical Talents - Specialist Program (grant number 2018-15), and the Multidisciplinary Cross Research Foundation of Shanghai Jiaotong University (grant number YG2019QNA28). The funders had no role in study design, data collection and analysis, decision to publish, or the preparation of the manuscript.

\section{Footnote}

Conflicts of Interests: The authors have no conflicts of interest to declare.

Ethical Statement: The authors are accountable for all aspects of the work in ensuring that questions related to the accuracy or integrity of any part of the work are appropriately investigated and resolved. All procedures performed in studies involving human participants were in accordance with the ethical standards of the institutional and/or national research committee and with the 1964 Helsinki declaration and its later amendments or comparable ethical standards. The study was approved by the Institutional Review Board (IRB) of Renji Hospital, School of Medicine, Shanghai Jiao Tong University \{IRB approval number: [2017]088\}. Informed consent was obtained from all individual participants included in the study.

\section{References}

1. Mariette C, De Botton ML, Piessen G. Surgery in esophageal and gastric cancer patients: what is the role for 
nutrition support in your daily practice? Ann Surg Oncol 2012;19:2128-34.

2. Migita K, Takayama T, Saeki K, et al. The prognostic nutritional index predicts long-term outcomes of gastric cancer patients independent of tumor stage. Ann Surg Oncol 2013;20:2647-54.

3. Liu X, Sun X, Liu J, et al. Preoperative C-Reactive Protein/Albumin Ratio Predicts Prognosis of Patients after Curative Resection for Gastric Cancer. Transl Oncol 2015;8:339-45.

4. Okadome K, Baba Y, Yagi T, et al. Prognostic Nutritional Index, Tumor-infiltrating Lymphocytes, and Prognosis in Patients with Esophageal Cancer. Ann Surg 2018. [Epub ahead of print].

5. Buzby GP, Mullen JL, Matthews DC, et al. Prognostic nutritional index in gastrointestinal surgery. Am J Surg 1980;139:160-7.

6. Onodera T, Goseki N, Kosaki G. Prognostic nutritional index in gastrointestinal surgery of malnourished cancer patients. Nihon Geka Gakkai Zasshi 1984;85:1001-5.

7. Mohri Y, Inoue Y, Tanaka K, et al. Prognostic nutritional index predicts postoperative outcome in colorectal cancer. World J Surg 2013;37:2688-92.

8. Nakatani M, Migita K, Matsumoto S, et al. Prognostic significance of the prognostic nutritional index in esophageal cancer patients undergoing neoadjuvant chemotherapy. Dis Esophagus 2017;30:1-7.

9. Pinato DJ, North BV, Sharma R. A novel, externally validated inflammation-based prognostic algorithm in hepatocellular carcinoma: the prognostic nutritional index (PNI). Br J Cancer 2012;106:1439-45.

10. Sun J, Wang D, Mei Y, et al. Value of the prognostic nutritional index in advanced gastric cancer treated with preoperative chemotherapy. J Surg Res 2017;209:37-44.

11. Siegel RL, Miller KD, Jemal A. Cancer statistics, 2018. CA Cancer J Clin 2018;68:7-30.

12. Mohri T, Mohri Y, Shigemori T, et al. Impact of prognostic nutritional index on long-term outcomes in patients with breast cancer. World J Surg Oncol 2016;14:170.

13. Morimoto LM, White E, Chen Z, et al. Obesity, body size, and risk of postmenopausal breast cancer: the Women's Health Initiative (United States). Cancer Causes Control 2002;13:741-51.

14. Kroenke CH, Chen WY, Rosner B, et al. Weight, weight gain, and survival after breast cancer diagnosis. J Clin Oncol 2005;23:1370-8.

15. Minicozzi P, Berrino F, Sebastiani F, et al. High fasting blood glucose and obesity significantly and independently increase risk of breast cancer death in hormone receptorpositive disease. Eur J Cancer 2013;49:3881-8.

16. Okada S, Shimada J, Kato D, et al. Clinical Significance of Prognostic Nutritional Index After Surgical Treatment in Lung Cancer. Ann Thorac Surg 2017;104:296-302.

17. McShane LM, Altman DG, Sauerbrei W, et al. Reporting recommendations for tumor marker prognostic studies (REMARK). J Natl Cancer Inst 2005;97:1180-4.

18. Ye LL, Oei RW, Kong FF, et al. The prognostic value of preoperative prognostic nutritional index in patients with hypopharyngeal squamous cell carcinoma: a retrospective study. J Transl Med 2018;16:12.

19. Zhao Y, Deng Y, Peng J, et al. Does the Preoperative Prognostic Nutritional Index Predict Survival in Patients with Liver Metastases from Colorectal Cancer Who Underwent Curative Resection? J Cancer 2018;9:2167-74.

20. Yang Z, Zhang B, Hou L, et al. Pre-operative prognostic nutritional index predicts the outcomes for triple-negative breast cancer. Tumour Biol 2014;35:12165-71.

21. Global BMI Mortality Collaboration, Di Angelantonio E, Bhupathiraju ShN, et al. Body-mass index and allcause mortality: individual-participant-data meta-analysis of 239 prospective studies in four continents. Lancet 2016;388:776-86.

22. Bhaskaran K, Dos-Santos-Silva I, Leon DA, et al. Association of BMI with overall and cause-specific mortality: a population-based cohort study of 3.6 million adults in the UK. Lancet Diabetes Endocrinol 2018;6:944-53.

23. Fontanella C, Lederer B, Gade S, et al. Impact of body mass index on neoadjuvant treatment outcome: a pooled analysis of eight prospective neoadjuvant breast cancer trials. Breast Cancer Res Treat 2015;150:127-39.

24. Chan DS, Vieira AR, Aune D, et al. Body mass index and survival in women with breast cancer-systematic literature review and meta-analysis of 82 follow-up studies. Ann Oncol 2014;25:1901-14.

25. Iyengar NM, Hudis CA, Dannenberg AJ. Obesity and cancer: local and systemic mechanisms. Annu Rev Med 2015;66:297-309.

26. Howe LR, Subbaramaiah K, Hudis CA, et al. Molecular pathways: adipose inflammation as a mediator of obesityassociated cancer. Clin Cancer Res 2013;19:6074-83.

27. Iyengar NM, Gucalp A, Dannenberg AJ, et al. Obesity and Cancer Mechanisms: Tumor Microenvironment and Inflammation. J Clin Oncol 2016;34:4270-6.

28. Sestak I, Distler W, Forbes JF, et al. Effect of body mass 
index on recurrences in tamoxifen and anastrozole treated women: an exploratory analysis from the ATAC trial. J Clin Oncol 2010;28:3411-5.

29. Jackisch C, Hegg R, Stroyakovskiy D, et al. HannaH phase III randomised study: Association of total pathological complete response with event-free survival in HER2positive early breast cancer treated with neoadjuvant- adjuvant trastuzumab after 2 years of treatment-free follow-up. Eur J Cancer 2016;62:62-75.

30. von Minckwitz G, Rezai M, Fasching PA, et al. Survival after adding capecitabine and trastuzumab to neoadjuvant anthracycline-taxane-based chemotherapy for primary breast cancer (GBG 40--GeparQuattro). Ann Oncol 2014;25:81-9.

Cite this article as: Wang Y, Battseren B, Yin W, Lin Y, Zhou L, Yang F, Wang Y, Sun L, Lu J. Predictive and prognostic value of prognostic nutritional index for locally advanced breast cancer. Gland Surg 2019;8(6):618-626. doi: 10.21037/gs.2019.10.08 